Chapter 13

\title{
HIV/AIDS: Vertical Transmission
}

\author{
Enrique Valdés Rubio \\ Additional information is available at the end of the chapter \\ http://dx.doi.org/10.5772/53204
}

\section{Introduction}

In The Eight Millennium Development Goals, the World Health Organization proposed to attempt at reverting the world negative HIV/AIDS epidemics through disease prevention and treatment for the year 2015. Attaining virtual elimination of Vertical Transmission (Prevention of mother-to-child transmission, PMTCT) worldwide is one of such aims [1].

The current chapter will address topics related to epidemiologic, pathophysiological, diagnostic and therapeutic aspects of vertical transmission of the Human Immunodeficiency Virus (HIV). Such information will enable the reader to understand how the health strategies aiming at preventing transmission to the fetus have turned into a paradigm of Perinatal Medicine, since the implementation of a series of biomedical interventions has proven to be successful to prevent transmission of HIV from an infected pregnant mother to her child.

\section{Epidemiology}

\section{World Epidemiological Status}

Prevalence of Vertical Transmission (VT) in the different regions of the world varies with geographic location and, specifically, with the economic resources invested by the different countries to support various strategies applied to health care policies that aim at the prevention and treatment of infected mothers. Proof of thereof is the fact that in countries with government programs in which the economic support is sufficient to implement the planned strategies for VT prevention, the prevalence of perinatal infection is under $2 \%[2,3]$.

HIV epidemics are showing a trend towards stabilization worldwide. Thus, in 2009 approximately 2.6 million persons became newly infected by the virus, representing $19 \%$ fewer than 
the newly infected in 1999 and more than a fifth less (21\%) of the estimates in 1997. In fact, in 33 countries the incidence of infection has fallen by $25 \%$. This includes 22 countries of the Sub-Saharan African region, the zone with the highest number of new cases worldwide (Ethiopia, Nigeria, South Africa, Zambia and Zimbabwe). In Eastern, Central and Western Europe, Central and Northern Asia the incidence of HIV infection has remained stable for the past five years. However in some high income countries infection rates have increased due to sex practices between homosexuals. Central Asia and Eastern Europe continue with high transmission rates among drug addicts and their sexual partners [4].

Regarding vertical transmission, an estimate of 370,000 children became infected with HIV during perinatal and lactation periods in 2009. Such figure is quite lower than the estimated 500,000 newly infected children in 2001. The latter has been possible thanks to Vertical Transmission (VT) prevention healthcare strategies that have been planned and implemented. Accordingly, the WHO and the UNAIDS proposed to virtually eliminate such transmission route by 2015 . To accomplish such goal, besides the efforts already implemented, they stated the importance of controlling the infection among youngsters. It is estimated that more than half of seropositive persons are girls and women [1,4].

In the Sub-Saharan African region, there are 8 times more infected women than men among persons aged between 15 and 24. Most of women are infected during unsafe heterosexual sexual activity. Such situation is the main responsible for the fact that such countries are assembling $90 \%$ of the children infected worldwide. Despite of the latter, the incidence of subjects with a carrier status among children under 15 years of age has declined in $32 \%$ in South Africa, the country in which AIDS is the main cause of maternal death and that accounts for $35 \%$ of deaths in children under 5 years of age. Accordingly, worldwide data show that only $15 \%$ of women in whom the carrier status had been detected during their perinatal period stayed subsequently on lifelong antiretroviral therapy [4].

It is estimated that approximately 4.9 million people in Asia were seropositive during 2009. Such figure is similar to those reported in the previous years, thus reflecting that the epidemics has stabilized. In such continent, the number of infected children under the age of 15 has increased marginally, from 140,000 in 2005 to 160,000 in 2009 and AIDS-related deaths have dropped in $15 \%$ since 2004 [4].

In Western Europe, epidemiology indicators regarding maternal carrier status have evolved favorably. The estimated prevalence of infection among pregnant women in the United Kingdom is $0.2 \%$ and HIV screening coverage in gestating women is $90 \%$. The latter has resulted in a sustained decline of VT from $12 \%$ in 1999 to $2 \%$ in 2007 [4,5].

In North America the carrier status has reached stability and VT has evidenced a dramatic decrease. In Canada, infection of the newborn has decreased from 5.2\% in 1997 to $2.7 \%$ in 2012, and when the seropositive mother was administered HAART, such figure reached $0.4 \%[4,6]$.

In Central and South America the number of affected children under 15 years of age is still low, with approximately 4000 new cases during 2009, in spite of the fact that the coverage of 
VT prevention programs is only marginally superior to that reported in countries with low or moderate income [4].

According to reports from 2006, the estimated number of seropositive subjects in Latin America is 1.7 millions, with 140,000 new cases and 65,000 deaths. Two thirds of the infected individuals live in four countries: Argentina, Brazil, Colombia and Mexico. The main agent responsible for the infection is HIV-1. The most commonly isolated genotype is type $\mathrm{B}$, followed by types $\mathrm{F}$ and $\mathrm{A}[2]$.

In the American countries that compose the Southern Cone, HIV/AIDS epidemiological surveillance is carried out through mandatory notification (passive mechanism) and in some, through active surveillance by means of sentinel centers [2].

Most of such Latin American countries have implemented, since the second half of the 90s, an Antenatal Prevention of Mother To Child Transmission (APMTCT) program, consisting of the early voluntary diagnosis of HIV infection in the gestating population, universal access to antiretroviral therapy (ART) for the mother-child binomial, the C-section surgery and suppression of lactation, a program that aims at eliminating vertical transmission as a route of infection $[2,3]$.

The main route of transmission in Latin America is represented by sex between men. There has been a significant increase in transmission through heterosexual intercourse since 1990. With the exception of Argentina, where initially transmission was mainly represented by needle sharing during drug injection, with a current dramatic drop, estimates of new cases of transmission through such route in 2005 do not exceed 5\%. On the other hand, there is concern in the area, regarding the relationship between HIV and drug addition, since it has been demonstrated that illicit drug abuse, regardless of the route of administration, favors risk behaviors [2].

In view of the above stated, it is possible to conclude that the features of the epidemics in the Southern Cone have varied, showing a trend to impoverishment, feminization, and shift towards homosexual and scholar populations. Moreover, the fact of the most affected age group is between 20 and 39 years is of great importance, since it demonstrates that the mostly affected population is that representing the childbearing age, a situation that has a direct impact on VT risk. The latter warrants the importance of implementing human sexuality education to all levels of the population, providing the necessary information and recognizing the right of women to be informed to enable them to take the appropriate measures to protect themselves and prevent HIV infection. [2-4].

The rate of HIV seropositive pregnant women fluctuates in the different countries of the Region, between 3 and 7/1000. It is estimated that more than 2 million HIV (+) women get pregnant and $90 \%$ of them belong to developing countries, thus between 370,000 and 500,000 infected children are born annually, that die mostly because of the disease [4]. Pregnancy would play a protective role for maternal mortality among HIV (+) women. Such effect would be related to the low pregnancy rates achieved among women at advanced stages of the disease. [2]. Conversely, in developed countries, the rate of maternal mortality has decreased significantly since the introduction of the Highly Active Antiretroviral Thera- 
py (HAART). Moreover, the latter has increased the probability of achieving pregnancy, thus decreasing the rate of stillbirths and gestation-related diseases [7].

Since the beginning of the pandemics, the importance of VT as transmission route has been clear. However, not even the most optimistic would have thought that PMTCT would turn into an example of effective biomedical intervention to prevent HIV transmission. It is important to point that most of Latin American countries have healthcare programs aiming at decreasing VT (PMTCT) that are based on the early prenatal voluntary screening for infection, the offering of antiretroviral therapies (ARVT), the C-section [8], substitution of breastfeeding [9] and multidisciplinary management during pregnancy. Regarding antenatal carrier status diagnosis, it is important to note that there are studies that reveal that between 25 and $55 \%$ of the total number of pregnant women who accept the test do not schedule a subsequent visit to know the results, thus they do not receive therapy. The latter suggests that fast intrapartum tests should be implemented on pregnant women unaware of their serologic condition. This is supported by a recent study carried out in Peru. The study concluded that the use of fast result tests (oral or blood) is a strategy advisable to decrease VT risk, in populations where PMTCT coverage does not reach the expected goals [10].

Pharmacological measures that have been implemented to decrease VT in the Southern region of America, were based on the ACTG 076 protocol [11] involving therapeutic antiretroviral management aiming at obtaining low or undetectable maternal viral loads, without toxic and/or teratogenic fetal effects. Such protocol consisted on the administration of Zidovudine to the mother during pregnancy and delivery, as well as to the newborn until the $6^{\text {th }}$ week of life, together with indication of an elective C-section operation and contraindication of breastfeeding. Such scheme managed to decrease VT in the region, from 29\% to 5.6\% during 2001 . [2] Subsequently, due to the emergence of new evidence supporting that triple-drug therapy was even more effective than monotherapy for VT prevention, a protocol was designed using a combination of reverse transcriptase and protease inhibitors. Such schemes were named together HAART. Thanks to such therapy, VT has dropped to figures close to $1 \%$ [2].

Because of the implementation and coverage of healthcare government programs in countries of the region, only few pregnant women have viral loads higher than 1000 copies $/ \mathrm{ml}$ in the proximity of delivery. The latter is responsible for the VT drop estimated as 1\%. [2] A recent study assessed the risk of VT in pregnant women who were administered PMTCT and concluded that the VT risk was 0.097 (95\% IC; 0.030-0.163). This reflects a reduction of transmission risk related to such route in almost $40 \%$ regarding VT before the implementation of such program [12].

Chile, where an early detection program and timely treatment are currently being carried out, is probably one of the countries in the region having reached the best VT rates. This is a result of healthcare strategies aiming an adequate and permanently updated diagnosis of the behavior of the epidemics in the country. The proportion of female HIV carriers has increased as compared to that of males, due to the fact that the main transmission route is sex (93.6\%). Such figure together with the impact of viral transmission both through homosexual as well as bisexual intercourse $(46.1 \%)$, positions the monogamous heterosexual woman as the highest risk population (51.1\%). Moreover, there is a sustained increase of cases trans- 
mitted through sex between men and women as compared to those transmitted through homosexual intercourse. This has shifted the epidemics towards heterosexual population groups, with a higher impact among female populations [2,3].

To summarize, HIV VT in the region is responsible for a low percentage of people reported with HIV/AIDS, but is accountable for most of the children affected by the infection in the Southern Cone. An important reduction of transmission by means of such exposition route, reaching levels very close to the proposed goals, has been evidenced. Such decline might probably be explained by the wide coverage and effectiveness of the measures propounded by the health authorities to tackle the issue $[2,3]$.

\section{Virology and Natural History of the Infection}

The Human Immunodeficiency Virus (HIV) is a lentivirus that belongs to the Retroviridae family. Two types of HIV have been identified: HIV-1, the most common, results in acquired immunodeficiency syndrome and death, and HIV-2, affecting mainly inhabitants or visitors from Western Africa, is transmitted less effectively and results in a more indolent disease with subsequent AIDS and death. They share less than 50\% sequence homology. Through phylogenetic analysis, HIV-1 strains can be divided into two large groups: The M (Major) group in turn divided into 10 subtypes (from A to J) and the $\mathrm{O}$ (Outlier) group. The eventual importance of HIV-1 typification lies in developing antibody screening techniques, diagnosing the infection in the newborn, and in the quantification of plasma viral RNA for follow up of infected patients [13, 14,15].

This monostranded RNA virus incorporates its genetic material into the host cell DNA, infecting cells that express CD4 antigen on their surface, such as helper T cells, macrophages, central nervous system cells and even placental cells $[13,16,17]$.

Once the infection has occurred, the patient may present a primoinfection characterized by mononucleosis-like non-specific symptoms, where antibody production is triggered. Subsequently the patient undergoes a silent carrier phase. The latter used to last between 9 and 11 years before the emergence of antiretroviral therapy. As the immune system is being progressively involved, the host becomes more susceptible to suffer opportunistic infections, cancers and dementia / encephalopathy, which are complications that enable labeling such stage as AIDS stage. In further consensus, the following findings were added: CD4 count under $200 / \mathrm{mm}^{3}$, uterine cervix cancer, lung tuberculosis and recurrent pneumonia $[13,16,17]$.

\section{Viral Replication Mechanism}

The genome of HIV-1 is relatively small, and composed of genes coding for structural, regulatory and accessory proteins. The viral particle joins the cells of the susceptible host, among which are T cells, monocytes, macrophages, follicular dendritic cells and microglial cells. A 
high affinity bond is generated between the gp 120 of the viral surface and the CD4 receptor molecule on the host cell. Cell membranes fuse together and the virus enters the cell, uncovering its RNA. It is propounded that after infection, it is the downregulation of CD4 expression on the infected cell surface that prevents a superinfection, enables an efficacious replication of the virus, and decreases the possibility of early cell death or apoptosis. During an early stage, the viral reverse transcriptase is activated together with other factors, and complete double stranded DNA copies are formed from the viral RNA. Such DNA copy is transported to the cell nucleus and binds to the host cell DNA through the viral integrase, generating the so-called provirus. The provirus may remain latent during a period of time. The host factors that influence the latency period duration are unknown. In activated cells, proviral transcription generates genomic RNA for its incorporation into new virions and messenger RNA that will be translated into structural proteins and several regulator and accessory proteins that facilitate viral replication, assembling and release $[13,16,17]$.

The adult immune system has several components that are critical for HIV infection, such as $\mathrm{B}$ and $\mathrm{T}$ cells, antigen-presenting cells, major histocompatibility antigens (types I and II), natural killer cells, cytokines and complement. Before an infection or antigenic stimulation, B cells are always "naïve". Following their stimulation, they generate two HIV specific subgroups that actively secrete antibodies against the virus. Likewise, there are "innocent" and other $\mathrm{T}$ cells with specific memory as a result of a prior exposure to the virus. In the great majority of the infected people HIV invasion disrupts normal immune reactions and induces a chronic progressive, polymorphic dysfunction that, at a given time, makes the individual susceptible to opportunistic infections, cancers, neurological disorders and premature death. The initial HIV infection in adults is frequently followed 2 to 6 weeks later by an acute viral syndrome that manifests with fever, pharyngitis, myalgia, tender lymphadenopathies, nonspecific rash and lasts from 7 to 14 days. There is an acute drop of circulating lymphocyte counts, with a fairly rapid normalization of figures. However, CD4+ T cell counts do not fully regain baseline values. During such period viral replication and dissemination to lymph nodes and mononuclear / macrophagic cells occur speedily $[13,16,17]$.

The circulating viral load is high in the initial absence of an effective antibody response. Typically, IgM appear one or two months after HIV exposure, followed by IgG (specifically anti-gp 120/41) and the development of an anti-p24 response that fades out in more advanced stages of the infection. Shortly afterwards, anti-gp120 and gp41 antibodies emerge, and persist for a lifetime. As anti-HIV antibody titers increase, the circulating viral load decreases and the infected subjects initiate an asymptomatic phase that commonly lasts from 3 to 11 years. During such asymptomatic phase viral replication is extremely intense, as well as is the destruction and replacement of CD4+ T cells. It should be taken into consideration that before causing a gradual CD4+ T cell depletion, HIV infection causes a CD4+ T cell dysfunction. Chemoprophylaxis, antibody-related cell-mediated cytotoxicity, intracellular antimicrobial activity and disorders of cytokine production also occur due to monocyte and macrophage dysfunction caused by the HIV infection [13,16,17].

During pregnancy, the mother undergoes a modulation of her immune response characterized by a TH2 response (humoral type) more prominent than TH1-type (cellular) response 
that renders her more susceptible to certain infections. Based on the latter, it has been postulated that pregnancy might influence the course of the infection. Such hypothesis has been thoroughly discussed, and currently the conclusion is that gestation does not have adverse effects on survival or on the expression of the disease. Such conclusion is supported by the fact that the absolute CD4 count and the viral load remain stable during pregnancy in untreated pregnant women $[7,13,16-18]$.

Almost all clinical disorders described in HIV-infected adults may appear in infants, however their rates of onset might be different in both life periods. The evolution of disease among infants infected during the perinatal stage is faster than in adults. This has raised questions such as if the developing immune system in fetuses and newborns represents a better ground for viral replication or is less efficient for infection control. Immaturity of the immune system of fetuses, newborns and infants causes two pathophysiological differences between adults and children: B cells are seldom susceptible to HIV infection and their circulating values remain normal; however such cells show abnormalities in infected children as compared to infected adults. As the infant has been exposed to few external antigens (and thus has a limited immune memory), the resulting immune dysfunction is more severe than in adults. Because of the poor development of $\mathrm{T}$ and $\mathrm{B}$ cell memory, children infected in perinatal stages are more vulnerable to antibody-mediated pyogenic infections than infected adults $[13,16,17]$.

Almost as soon as the infected cells die, they are replaced, until the degree of the immune dysfunction is such as to make the immune control of the virus inefficient. Thus, the clinical conditions defining AIDS are progressively met.

\section{HIV behavior in women}

The main HIV transmission risk factor for females worldwide is sexual intercourse. More than $90 \%$ of HIV-AIDS-infected women have acquired the infection through heterosexual transmission. In most of the cases, such women had sexual intercourse only with their stable spouse, thus attesting for their vulnerability to transmission, which is directly related to their sexual partner's behavior (bisexuality, drug addictions, promiscuity) [3].

The reasons explaining why women are more susceptible to be infected by HIV than men are varied. Thus, healthcare staff must be aware of such factors and therefore generate a sympathetic and empathic environment to achieve a good physician-patient relationship. Among such reasons, the following should be considered:

- Sexual transmission of the virus is several times more common from men to women than vice versa. Women with thinned vaginal mucosa or lacking the physiological defense mechanisms (pubertal and postmenopausal women) are at higher risk.

- Asymptomatic sexually transmitted vaginosis is commonly underdiagnosed and results in a disturbance of the indemnity of the vaginal mucosa. 
- Women depend economically, socially and emotionally on men and such situation makes rejection of high-risk sexual intercourse difficult.

- Cultural norms encourage promiscuity among males, thus increasing transmission risk among monogamous females.

- There is a lack of easy access to woman-controlled-preventive methods (female condoms).

Regarding the last point and aiming at identifying other prevention methods, recent studies have demonstrated that the use of tenofovir gel might be a useful tool to prevent infection. In fact, a South African study concluded that the pre and post-coital prophylactic use of such microbicide among women aged between 18 and 40 reduced HIV infection in 39\% and HSV-2 infection in 51\%. [19] On the other hand, circumcision in adults has proven to be effective to prevent infection among seronegative males with an HIV+ partner. The latter has led the UNAIDS and WHO to recommend such intervention in countries with high prevalence [4]. Finally, there are also studies that propose the use of tenofovir prior to sexual intercourse in infected or high-risk couples. All these prophylactic measures should be assessed with further adequately designed studies.

Probably due to such reasons and in light of the pooled data in developing and developed countries, the proportion of HIV carrier women has increased as compared to that of men. The cause is that the main transmission route is sex. The latter together with the impact of viral transmission route in homo and heterosexual mode, have transformed heterosexual monogamous women into the highest risk population. Moreover, there is a sustained increase of cases transmitted by sexual intercourse between men and women as compared to those transmitted through homosexual intercourse. This has shifted the epidemics towards population groups with a lower social-economic level and with a clear decrease of females in higher school education levels [2, 3, 16,17].

Epidemiology studies suggest that the observed prevalence among women in childbearing age is comparable to prevalence in pregnant women. On the other hand, pregnancy rates in seropositive women that have not developed AIDS are comparable to those seen among uninfected women, while among women who developed the disease the probability of getting pregnant is quite lower [13-15].

\section{Management of seropositive pregnant woman for Vertical Transmission Prevention}

Most of women discover their carrier status or their disease during pregnancy or subsequently after birth upon screening of their offspring. With regard to the need for screening of all women during their pre and/or post-conception consultation, the American College of Obstetricians and Gynecologists recommends carrying it out on a routine basis, which is a conduct commonly adopted in many countries of the world. In fact, in most of these countries, such screening is performed together with the mandatory pre-test counseling act, with the informed consent and with the willfulness of individuals in the decision to undergo the 
test. Therefore, screening requires the participation of staff trained in "counseling", which has reinforced the decision to undergo the test among women. This has shown to be of most importance for the generation of awareness about the disease, adherence to therapy and the incorporation of behaviors by the carrier to prevent transmission to her personal environment [20-24]. Pre and post-conceptional counseling is understood as a "confidential dialogue between a questioner and a counselor aiming at empowering women to face stress, decision making regarding HIV/AIDS during pregnancy and discussion of the elements for prevention of vertical transmission". It is important to recommend the following topics to be addressed upon counseling:

- Impact of HIV on pregnancy.

- Vertical Transmission risk and impact of prophylactic measures.

- Risks and benefits of antiretroviral therapy.

- Prognosis for children who become infected.

- Breastfeeding-related risks.

\section{Diagnosis}

The most popular methods worldwide are tests that screen for specific antibodies against viral antigens. The most used technique for screening is Enzyme Linked Immunosorbent Assay (ELISA). The most used technique for infection confirmation is the Western blot technique [25].

The ELISA test uses antigens derived from the intact virus and binds them to microtitration wells. The serum or plasma from the patient is added into the wells and if there are antibodies against HIV they bind to the antigen present. A chromogen substrate is also provided to evidence the potentially bound enzyme and the intensity of the color generated is read on a spectrophotometer. Color reading is proportional to the amount of the enzyme-antibody complex that bound the HIV antigen present in the wells. Commercially available tests have sensitivity and specificity rates between 98 and 100\%. The disadvantage of such tests is they detect antibodies and thus may yield false positive results if transmission occurred shortly before the test. Such time lapse is known as window period, which may last between 3 and 6 months. For such reason, fourth generation ELISA is recommended. Such test is able to detect simultaneously Ag p24 and its respective antibodies, and therefore it can shorten the window period to approximately 30 days, with $99.9 \%$ sensitivity and $99.5 \%$ specificity. Despite the latter, it is important to point that the positive predictive value of this test during pregnancy is approximately $50 \%$, because it is applied to a low prevalence population, and therefore a confirmation test is mandatory (Western blot) [16].

Additionally, in pregnant women with unknown serology status and attending consultation for labor or medical situations where pregnancy termination is imminent, services should have rapid diagnostic techniques. Despite their non-optimal specificity and sensitivity, posi- 
tive results on such tests should be able to generate the recommendation of preventive measures during the peripartum period, with prior patient informed consent $[5,10]$.

The confirmation Western blot technique is carried out with a nitrocellulose strip to which HIV shell proteins are added. Patient serum is applied on the strips: any antibody against such virus present in serum will bind to its specific antigen. This generates a series of dark bands. By comparing the band position with a control, it is possible to determine if the patient's blood contains HIV-specific antibodies [16].

On occasions, individuals with recent infection or in the process of structuring a complete antibody response yield undetermined responses. In such cases, the test should be repeated within one to two months.

PCR tests are sensitive and useful to confirm the infection status. They are able to detect very small amounts of virus and they do not rely on an antibody response to infection. PCR is used in clinical practice to establish the infection status on infants born to infected mothers and potentially within the window period between infection and presence of detectable specific antibodies. PCR and other amplification techniques have been improved to quantify HIV RNA. There is considerable interest on perfecting reliable tests to detect the virus in oral fluids or urine, since they are easy to collect without causing either disturbance to the patient or risks to the collector. Moreover, they can be especially helpful in children and adults in whom vein access can be difficult [16].

Assessment of HIV infection status is complex in children under the age of 6 months because of gestation and early lactation immunology. The most commonly used tests in adults are based on the detection of IgG antibodies against the virus. During pregnancy, maternal IgG crosses easily the placenta towards fetal circulation, where it remains until 18 months after birth. For such reason, assessment of the HIV infection status in infants requires testing other than ELISA and Western blot. The options are virus cultures and PCR, as well as detection of IgA antibodies directed against HIV [14,16].

\section{HIV and Pregnancy}

According to epidemiological studies, pregnancy rates among seropositive women that have not presented AIDS are comparable to rates in uninfected women. The probability of becoming pregnant is quite lower among women who develop AIDS. Despite of certain contradictory evidence, latest studies seem to confirm that pregnancy does not affect the course or the complications of the disease [26].

As for the role played by the viral load or the immune condition of the pregnant woman, there is evidence supporting a close relationship between high viral loads and higher VT risk. However there is not a viral load warranting a virus-free neonate. Mother-to-child transmission is possible despite viral levels being undetectable in the mother. On the other hand, maternal CD4 concentrations $<700$ cells/mm relate to a higher VT risk [27]. 
Nevertheless, opportunistic infections (Pneumocystis jirovecii, Herpes Zoster, etc.) and prevalent diseases such as tuberculosis and malaria are those with more aggressive course in seropositive pregnant women and increase significantly maternal and perinatal morbidity and mortality. [28]

Vertical transmission is one of the mostly studied transmission routes, thus it is possible to share some conclusions regarding the moment of transmission to the fetus or newborn and the factors that increase VT risk $[14,16]$.

Moment of transmission to the product of conception:

- During pregnancy: up to $35 \%$.

- During delivery: up to $65 \%$.

- During lactation: up to $14 \%$.

Obstetric factors that favor VT:

- Premature Rupture of Membranes of more than 4 hours.

- Premature delivery.

- Low Birth Weight (under 2,500 g).

- Ovular infection.

- Direct contact of the fetus with cervical and vaginal secretions and/or blood in the birth canal.

Fortunately, the gravid status does not seem to have an impact on the course or the natural history of HIV infection, since although a decrease in CD4+ T cell counts has been described during pregnancy, they return to baseline values after delivery [17]. In the absence of complications such as drug addictions or chronic medical diseases, the incidence of obstetric pathology does not increase; spontaneous abortion, intrauterine growth restriction, preterm delivery and stillbirth are within the expected prevalence range. However, the risk of infectious complications increases among pregnant women with a significant impairment of the immune system $(\mathrm{CD} 4<300 / \mathrm{dl})[18]$.

A seropositive gestating woman should be clinically addressed based on a multidisciplinary and thorough assessment of her initial health status. The latter should include a full physical examination, especially observing those signs that guide diagnosis towards an opportunistic infectious pathology and assessing the current immune status. Upon indicating the therapeutic and/or prophylactic antiretroviral treatment, a risk versus benefit assessment should be carried out to evaluate the effectiveness of the pharmacological scheme and the eventual teratogenic and toxic effects on mother and fetus.

The following laboratory tests might be performed in addition to the routine tests that should be requested for pregnant women at the beginning of their prenatal follow-up $[4,16]$ :

Infectious parameters: 
- Rubella serology

- Urine culture

- Hepatitis B surface antigen Hepatitis B core antigen for Hepatitis B virus.

- Serology for the detection of Syphilis (RPR or VDRL).

- Serology for Hepatitis C.

- PPD (skin test for tuberculosis).

- Serology for certain parasitoses depending on their geographic prevalence (Toxoplasma gondii, Chagas disease, Malaria, etc.).

- Serology for cytomegalovirus.

- Culture for gonococcus, Chlamydia, Mycoplasma and Ureaplasma.

Immunological parameters:

- CD4+ T cell counts

- Viral load assessment (PCR).

Sequential detection of the viral load and CD4 counts are used to predict the risk of a rapid disease progression in the pregnant woman and the eventual VT in such patient group. However, as an intent to identify a more cost-effective test than the latter, the role of HIV typification through sequential study of specific bands offered by the Western blot technique has been studied recently. Such preliminary studies demonstrated that the absence of anti pol antibodies was associated with an acute infection. Although it is true that the lack of anti gag p39 antibodies was related to a rapid disease progression and a higher probability of infection of the newborn, such absence was not as statistically significant to predict a higher risk for VT. Therefore, such diagnostic tool needs still to be upheld by further studies before it becomes indicated for clinical use. [29]

All antenatal diagnostic assessments may be carried out without contradictions, except for those involving invasive techniques that imply an additional infection risk to the fetus (chorionic villus biopsy, amniocentesis, cordocentesis).

\section{Antiretroviral therapy during pregnancy}

The aim of antiretroviral therapy during pregnancy is to decrease the maternal viral load to undetectable levels without causing deleterious or teratogenic effects on the product of conception, and on the other hand, to decrease the risk of vertical transmission. To attain such goal, there are currently 14 antiretroviral drugs available (Table 1). Such drugs must be used within schemes individually adapted for each patient. Drug selection should be based on the prior treatment of the woman (if she were under any), the current status of the patient and her motivation, the viral load, drug resistance, CD4+ T cell counts, and associated toxic 
and teratogenic effects (Table 2). The use of Zidovudine is recommended within the scheme. Zidovudine has been the only drug to enter a protocol and that has demonstrated efficacy in the protection of the fetus from vertical transmission. However, new studies have demonstrated the efficacy of other antiretroviral drugs in VT reduction.

\begin{tabular}{lc}
\hline \multicolumn{1}{c}{ Generic name } & FDA Classs \\
\hline Abacavir & $\mathrm{C}$ \\
\hline Didanosine (ddl) & $\mathrm{B}$ \\
\hline Lamivudine (3TC) & $\mathrm{C}$ \\
\hline Lamivudine + Zidovudine (Convivir) & $\mathrm{C}$ \\
\hline Stavudine (d4T Zalcitabine) & $\mathrm{C}$ \\
\hline Zidovudine (ZDZ, AZT) & $\mathrm{C}$ \\
\hline \multicolumn{1}{c}{ Non-nucleosidic reverse transcriptase Inhibitors } \\
\hline Delavirdine & $\mathrm{C}$ \\
\hline Efavirenz * & $\mathrm{X}$ \\
\hline Nevirapine & $\mathrm{C}$ \\
\hline Amprenavir * & \\
\hline Indinavir & $\mathrm{X}$ \\
\hline Nelfinavir & $\mathrm{C}$ \\
\hline Ritonavir & $\mathrm{X}$ \\
\hline Saquinavir & $\mathrm{B}$ \\
\hline
\end{tabular}

Table 1. ANTIRETROVIRAL AGENTS,Nucleosidic reverse transcriptase inhibitors

\begin{tabular}{l}
\hline A: Controlled studies show no risk \\
\hline B: No evidence of risks in humans \\
\hline C: Risks cannot be ruled out. However, a potential benefit might justify its use. \\
\hline D: Positive evidence of risk. \\
\hline X: Contraindicated in pregnancy.
\end{tabular}

Table 2. FDA Drug Category Rankings (Drugs and pregnancy)

\section{Management Protocol in the Prevention of Vertical Transmission (ACTG 076)}

The first formalized intent in prevention of VT was that assessed by a joint study carried out in France and the United States, attempting at the evaluation of a management protocol (Clinical Group Protocol 076-ACTG 076) [11], consisting of a study and follow up of HIV+ pregnant women that had a gestational age between weeks 14 and 34, without antiretroviral therapy or indications for such therapy, with CD4+ T cell count $>$ or equal to 200 cells $/ \mathrm{mm}^{3}$. 
The group that was administered placebo was compared to the group receiving AZT (orally during pregnancy, injectable during delivery and as syrup to the newborn for 6 weeks). Results showed that transmission was $8.3 \%$ with AZT and $25 \%$ with placebo, which is equivalent to a reduction in $67 \%(p=0,00006)$. (Table 3$)$

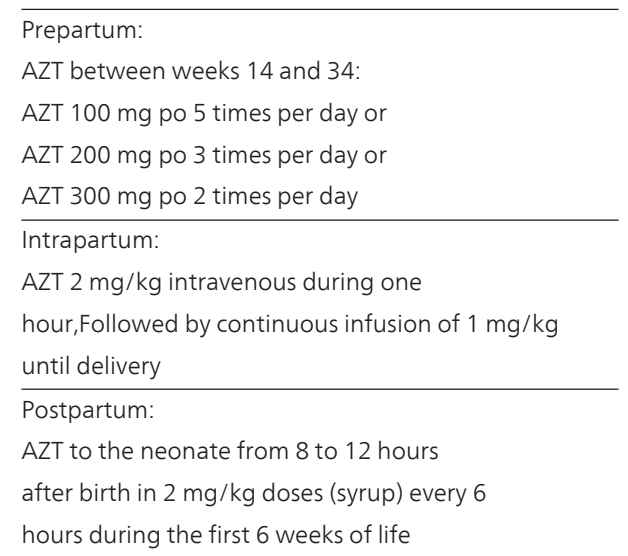

Table 3. ACTG 076 PROTOCOL

\section{High Activity Antiretroviral (ART) Therapy (HAART)}

The first attempt at a successful pharmacological scheme to control VT was achieved by the ACTG 076 protocol. Such protocol together with elective C-section and suppression of lactation (biomedical measures) achieved a decrease in VT from $29 \%$ to 5.6\% [11]. Although the latter is true, new evidence demonstrated that the triple drug therapy was more effective than monotherapy or bitherapy to prevent Vertical Transmission (VT). A protocol, that combined the biomedical preventive measures already mentioned and the indication of three antiretroviral drugs was designed. Such drugs included nucleosidic and non-nucleosidic reverse transcriptase inhibitors (NRTI and NNRTI respectively) and Protease inhibitors (PI), and together formed a series of schemes known as highly active antiretroviral therapy (HAART). It is by virtue of the latter that VT has decreased to values close to 1.0 [2-6, 30-34].

It is a known fact that VT may occur among seropositive pregnant women during pregnancy $(35 \%)$, delivery $(65 \%)$ and lactation $(14 \%-29 \%)$ and that there are factors that increase the risk of fetal infection (Primoinfection, Sexually transmitted diseases and CD4+ T cell counts). The most important of such factors is maternal viral load [27]. Loads under 1000 copies $/ \mathrm{ml}$ have significantly lower risks of $\mathrm{VT}$, however there is no evidence pointing to the minimal load that exempts from risk. The latter supports the need to use a highly active antiretroviral therapy (HAART) with the highest effectiveness and safety to reach, hopefully, undetectable viral load levels. With such purpose, and considering that most of transmissions to the newborn occur during the peripartum period, an attempt at reaching the lowest viral loads dur- 
ing the third trimester of gestation (weeks 34-36) should be made. However, due to the potential toxic effects of therapeutic drugs, it is suggested their use is limited to the periods of maximum effectiveness considering risks and benefits of such exposure to the motherchild binomial. Anyway, AZT should be added to the therapeutic scheme if possible, even in cases in which such drug was not used during the antepartum period, since it has proven to be helpful to decrease vertical transmission even in abbreviated schemes (intrapartum and postpartum) $[4,5,34]$.

Based on the above-mentioned, HAART type ART should be indicated in the following situations: [34]

1. In seropositive pregnant women without prior treatment from week 24

2. In pregnant women with viral loads higher than 100,000 copies/ml from 14 weeks

3. In case of seroconversion during pregnancy it should be indicated at once

Within the HAART concept, the selection of the NRTI combination to be used should follow the efficacy of the latter in reducing VT, considering the potential teratogenic effects and the eventual toxicity to the mother-child binomial. Based on the teratogenesis risk classification of the FDA, NRTI are classed as B or C. The largest experience involves Zidovudine (AZT or ZDV) that has demonstrated being quite a safe drug, with low resistance and high efficacy to prevent VT. In addition, the association of ZDV and Lamivudine (ZDV/3TC) has demonstrated to be more effective than ZDV as monotherapy in mother-to-child transmission prevention. Such combination does not imply higher toxicity or risk of teratogenesis [2-6, 30-34].

Regarding the search for the third drug, Lopinavir-Ritonavir has demonstrated efficacy and safety in VT prevention. The association of Saquinavir reinforced with Ritonavir has gathered enough evidence to suggest that such combination is a good supplementary alternative to render the pharmacological therapy more effective [26].

Although Nevirapine (NVP) has been widely indicated and has demonstrated being effective in the prevention of mother-to-child transmission mostly in developing countries with low economical resources, the use of such drug has been related to the development of viral resistance both in mothers as in children who were infected despite the received prophylaxis. Moreover, mothers who were administered Nevirapine had a higher incidence of toxic effects (hepatic, hypersensitivity) in particular pregnant women with CD4+ T cell counts higher than 250 cells/ml. The above mentioned suggests that such indication should be considered among patients never having received Nevirapine and with CD4+ T cell counts under 250 cells $/ \mathrm{ml}$. When such drug is used in the intrapartum period, it is advisable to indicate ZDV y Lamivudine (3TC) for 7 days both to the mother as well as to the newborn, to reduce the risk of viral resistance to Nevirapine and other NNTRI [30-34].

The following are among the drugs that should not be indicated during pregnancy: Efavirenz, Nelfinavir and the association of d4T (Stavudine) and ddI (Didanosine). All share teratogenicity and toxicity risk for the mother-child binomial [30-36].

In 2010 the World Health Organization stressed the importance of offering a lifelong antiretroviral therapy to patients with a carrier condition recognized during pregnancy. The 
WHO proposed two prophylactic treatment schemes (A and B) for pregnant women who did not meet the criterion of a certain CD4+ T cell count $\left(<350 / \mathrm{mm}^{3}\right)$. A third option $(B+)$ as appeared recently, aiming at implementing the HAART for a lifetime to all seropositive pregnant women regardless of their CD4+ T cell count (Table 4). This new option has the following advantages: it simplifies drug supply, since it relates drugs to national antiretroviral therapy programs, protects against VT, prevents transmission to seronegative partners and prevents discontinuation of the follow up of therapies already implemented by the patients. It finally suggests that such new option should be evaluated depending on the reality of each country [31].

\begin{tabular}{|c|c|c|c|}
\hline & \multicolumn{2}{|c|}{ Woman Receives: } & \multirow{4}{*}{ Infant receives: } \\
\hline & Treatment & Prophylaxis & \\
\hline & (for CD4 count & (for CD4 count & \\
\hline & $\leq 350$ cells $/ \mathrm{mm}^{3}$ ) & > 350 cells $/ \mathrm{mm}^{3}$ ) & \\
\hline \multirow[t]{7}{*}{ Option Aa } & \multirow{7}{*}{$\begin{array}{l}\text { Triple ARVs starting as soon as } \\
\text { diagnosed, continued for life }\end{array}$} & \multirow{4}{*}{\multicolumn{2}{|c|}{$\begin{array}{l}\text { Antepartum: AZT starting as early Daily NVP from birth until } 1 \\
\text { as } 14 \text { weeks gestation week after cessation of all } \\
\text { Intrapartum: at onset of labour, breastfeeding; or, if not } \\
\text { single-dose NVP and first dose of breastfeeding or if mother is }\end{array}$}} \\
\hline & & & \\
\hline & & & \\
\hline & & & \\
\hline & & $\mathrm{AZT} / 3 \mathrm{TC}$ & on treatment, through age \\
\hline & & Postpartum: daily AZT/3TC & $4-6$ weeks \\
\hline & & through 7 days postpartum & \\
\hline \multirow[t]{7}{*}{ Option $B_{a}$} & Same initial ARVs for both & Same initial ARVs for both: & Daily NVP or AZT from birth \\
\hline & & Triple ARVs starting as early as 14 & 4 through age $4-6$ weeks \\
\hline & Triple ARVs starting as soon as & weeks gestation and continued & regardless of infant feeding \\
\hline & diagnosed, continued for life & intrapartum and through & method \\
\hline & & childbirth if not breastfeeding or & \\
\hline & & until 1 week after cessation of all & \\
\hline & & breastfeeding & \\
\hline \multirow[t]{5}{*}{ Option B+ } & Same for treatment and & Same for treatment and & \multirow{5}{*}{$\begin{array}{l}\text { Daily NVP or AZT from birth } \\
\text { through age } 4-6 \text { weeks } \\
\text { regardless of infant feeding } \\
\text { method }\end{array}$} \\
\hline & prophylaxis: & prophylaxis: & \\
\hline & Regardless of CD4 count, triple & Regardless of CD4 count, triple & \\
\hline & ARVs starting as soon as & ARVs starting as soon as & \\
\hline & diagnosed,c continued for life & diagnosed,c continued for life & \\
\hline
\end{tabular}

Table 4. Note: "Triple ARVs" refers to the use of one of the recommended 3-drug fully suppressive treatment options. For the drug abbreviations in the table: AZT (azidothymidine, zidovudine [ZDV]); NVP (nevirapine); 3TC (lamivudine).a Recommended in WHO 2010 PMTCT guidelines b True only for EFV-based first-line ART; NVP-based ART not recommended for prophylaxis (CD4 >350) c Formal recommendations for Option B+ have not been made, but presumably ART would start at diagnosis. Options for PMTCT programs 


\subsection{Toxic and Side effects of ART}

The eventual toxic effect of such medications on the mother-child binomial is among the aspects that need to be assessed for the election of the pharmacological treatment. According to study reports, approximately $80 \%$ of gestating women under treatment developed some side effect such as anemia, nausea, vomiting, hepatic enzyme disorders or hyperglycemia [26,35-37]. Because of this, it is important to know which are the side and toxic effects of the drugs that are commonly used, in order to identify them and treat them accordingly.

\subsection{Protease inhibitors and hyperglycemia}

In non-pregnant women, the use of protease inhibitors has been related to the development of different degrees of carbohydrate Intolerance. This should be considered when they are used during pregnancy since they may trigger gestational diabetes [26,35-37].

\subsection{Mitochondrial toxicity and nucleosidic reverse transcriptase inhibitors}

Reverse transcriptase nucleoside inhibitors are recognized for their induction of mitochondrial dysfunction due to their affinity for mitochondrial DNA-polymerase gamma. Such effect is more intense for Stavudine (d4T), ddI (Didanosine) and less intense for ZDV (Zidovudine), 3TC (Lamivudine), ABC (Abacavir) and TDF (Tenofir). Additionally, these drugs are also related to lactic acidosis in cases with concomitant hepatic steatosis. Such association would be higher with the use of d4T (Stavudine) with an estimated rate of $0,8 \%$ and $1,2 \%$ per year per treated patient. [26, 35-37]. Clinical manifestations of this condition are varied and include polyneuritis, myopathies, cardiopathies, hepatic steatosis and lactic acidosis. Not uncommonly patients develop a condition similar to the HELLP Syndrome that should be considered when addressing differential diagnoses. Finally, there are reports about uninfected children, born to seropositive mothers that received ZDV or ZDV/Lamivudine (ZDV/3TC) during pregnancy, who developed mitochondrial dysfunction -related symptoms during the first months of life. Such finding was not confirmed with the protocol assessment ACTG 076 [11].

Regarding the mode of delivery in seropositive pregnant women who did not receive ART or had partial indication of ART, elective C-section at week 38 (a surgical procedure carried out before the initiation of labor and without premature rupture of membranes) has demonstrated a decrease of VT risk in one half. $[8,38]$. Recent studies propose the option of vaginal delivery to be used according to the obstetric conditions in seropositive women with viral load under 1000 copies/ml and prior patient consent. Such indication is supported by the fact that C-section benefits are indeed difficult to evaluate and moreover, data retrieved from a large number of patients did not evidence a higher reduction of VT among patients with viral loads < 1000 copies/ml that underwent C-section. Furthermore, C-section increased infectious morbidity between 7 and 10 fold [33].

When facing the vaginal delivery option, episiorrhaphy and forceps or spatulas should be avoided. Invasive procedures such as amniocentesis, chorionic villus biopsy and invasive monitoring might increase fetal exposure to infected maternal blood, and thus should also 
be avoided. The use of oxytocin has no contraindications, as opposed to ergot-derivatives that might present an eventual synergic action in association to Protease Inhibitors that would exaggerate vasoconstriction and ischemia, and therefore are contraindicated [39].

During delivery (C-section or vaginal delivery), the indication of intravenous administration of ZDV to the mother should be the rule, since fetal plasma levels of such drug, reached by transplacental route, ensure an adequate pre-exposition prophylaxis. Such indication together with the administration of ZDV suspension orally to the newborn during six weeks increases the preventive effect on infection transmission. This is regardless of the mother being administered ZDV within the therapeutic scheme or even in the event of resistance to ZDV. Finally, the use of Nevirapine (200 mg one time only dose) should only be indicated to patients with late initiation of the prevention protocol, on patients with a viral load $>1000$ copies/ml or in the case of diagnosis of maternal carrier status during labor $[4,5,33,34]$.

There is evidence pointing that among patients that are not treated within an antiretroviral management protocol, transmission to the newborn through breastfeeding ranges between $5 \%$ and $14 \%[9,40]$. Free and cell-bound virus has been isolated from breast milk through PCR techniques and viral culture. Such cells are more numerous in the colostrum and in breast milk secreted during the immediate puerperium. The latter, in association with the immature immune system of the newborn, make transmission through breastfeeding higher during the first month of life. All the above-mentioned, warrant the recommendation to suppress lactation in countries where infantile malnutrition and diarrheic syndrome - related mortality are low [34].

\section{Summary of Mother-to-child Prevention Management}

1. Every pregnant woman should be offered the possibility of undergoing HIV testing not later than upon the second pregnancy follow-up visit within the first trimester of gestation, and depending on resources, also within the third trimester.

2. A quick HIV test for HIV diagnosis enabling immediate action to be taken should be offered to women who arrive to delivery without having their test results available, even if tests have been carried out. Counseling and consent are mandatory.

3. ART is recommended to prevent HIV vertical transmission in pregnant women without prior therapy between gestation weeks 20 and 28. The use of ZDV in association with Lamivudine is recommended.

4. Viral loads should be followed up 6 weeks after initiation of ART during gestation and subsequently on a monthly basis until gestation weeks 34 and 36 .

5. Elective C-section should be indicated at 39 weeks in HIV+ women without ART over pregnancy, in women that do not have a viral load result upon weeks 34-36, in those with a viral load $>1.000$ copies/ $\mathrm{ml}$ or in women co-infected with Hepatitis C virus.

6. In the event a pregnant woman meets the conditions for a vaginal delivery, her consent should be obtained after being informed about the eventual associated risks. Epis- 
iorrhaphy and all procedures involving the use of instruments should be avoided (forceps or spatulas).

7. Intrapartum intravenous ZDV should be used regardless of the delivery mode chosen.

8. ZDV suspension should be administered orally for 6 weeks starting from 8 to 12 hours from birth, to all newborns to HIV+ mothers. The recommended ZDV dose for term newborns is $2 \mathrm{mg} / \mathrm{Kg} /$ dose every 6 hours p.o. or $1.5 \mathrm{mg} / \mathrm{Kg} /$ dose every 6 hours intravenous. The same dose should be administered every 12 hours in preterm newborns of less than 35 weeks gestational age.

9. If Nevirapine is administered intrapartum, ZDV/3TC must be associated for 7 days after delivery to reduce the risk of developing NVP resistance.

10. Lactation should be suppressed with cabergoline or bromocriptin.

11. The newborn should be administered ZDV suspension, orally, for 6 weeks starting from 8 to 12 hours after birth. The recommended AZT dose for term newborns is $2 \mathrm{mg} / \mathrm{Kg} /$ dose every 6 hours orally or $1.5 \mathrm{mg} / \mathrm{Kg} /$ dose every 6 hours intravenous. The same dose should be administered every 12 hours in preterm newborns of less than 35 weeks gestation.

12. Newborns to mothers who received NVP as part of mother-to-child transmission prevention should receive, besides ZDV, 2 doses of $2 \mathrm{mg} / \mathrm{Kg}$ of NVP oral solution. The first dose should be administered the earliest as possible postpartum and the second should be administered at 48 to 72 hours of life.

13. The newborns to mothers who did not receive the mother-to-child transmission prevention protocol, or who just received intrapartum prophylaxis, should be administered the AZT scheme and 1 to 2 NVP doses.

\section{Conclusion}

A seropositive gestating woman should be clinically addressed based on a multidisciplinary and thorough assessment of her initial health status. The latter should include a full physical examination, especially observing those signs that guide diagnosis towards an opportunistic infectious pathology and assessing the current immune status.Once the effectiveness of biomedical healthcare provisions for the prevention of MTCT (mother-to-child-transmission) is demonstrated, it is important to ensure collection of epidemiological history in order to achieve an adequate report, key data to reassess the design and the effectiveness of preventive programs. To achieve such aims, it is critical to maintain and improve diagnosis and primary prevention of the infection among women in childbearing age. ON the other hand, it is important to prevent high rates of unwanted pregnancies and abortions that are direct indicators of risk behaviors in such population group. Likewise, one should aim at achieving $100 \%$ screening during the first trimester, also at the possibility of repeating it during the third trimester and at training maternity staff on rapid testing for carrier status detection 
during labor, for those pregnant women without having accessed an AMTCPP (Antenatal Mother-to-Child Prevention Program).

Finally, MTCT prevention strategy has been based on the ongoing revision of the pooled evidence, therefore it is of key importance to continue evaluating new conducts that enable identification of other aspects: the eventual induction of antiviral resistance and toxicity on the pregnant woman and the newborn and their potential impacts on further quality of life, the use of micronutrients and their impact on MTCT decrease, assessment of the vaginal delivery option in gestating women with low viral load, and sperm wash as an element to reduce MTCT [40].

\section{Nomenclature}

VT: Vertical Transmission:

HIV: Human Immunodeficiency Human

AIDS: Acquired Immune Deficiency Syndrome

PMTCT: Prevention of mother-to-child transmission

AMTCPP: Antenatal Mother-to-Child Prevention Program

UNAIDS: Joint United Nations Program on HIV/AIDS

ART: Antiretroviral Therapy

HAART: Highly Active Antiretroviral Treatment

NRTI: Nucleoside reverse transcriptase inhibitor

NNRTI: Non nucleoside reverse transcriptase inhibitor

PI: Protease inhibitor

AZT or ZDV: zidovudine

NVP: Nevirapine

3TC: Lamivudine

d4T: Stavudine

ddI: Didanosine 


\section{Author details}

Enrique Valdés Rubio*

Address all correspondence to: evaldes@vtr.net

Maternal -Fetal Medicine Unit. Obstetrics and Gynecology Department. Hospital Clínico Universidad de Chile. Santiago, Chile

\section{References}

[1] The Millennium Development Goals. (2012). Eight goals for 2015. Found in: http:// www.who.int/topics/millennium_development_goals/diseases/es/index.html Access on June $30^{\text {th }}$.

[2] Valdés, E., Candia, P., \& Lattes, K. (2009). Transmisión Vertical de VIH y SIDA: Realidad epidemiológica del Cono Sur Progr Obstet Ginecol. 52(12), 719-24.

[3] Valdés, E., Sepúlveda, A., Candia, P., Sepúlveda, C., \& Lattes, K. (2011). VIH/SIDA: Comportamiento epidemiológico de la transmisión vertical en el contexto general de la infección en Chile. Rev Chil Obstet Ginecol, 76(1), 52-57.

[4] UNAIDS. (2010). Global Report. Found in: http://www.unaids.org/globalreport/global_report.htm Access on June 30 2012.

[5] Royal College of Obstetricians and Gynaecologists. (2010). Management of HIV in pregnancy. Green-top Guideline N`39 June. Found in: http://www.rcog.org.uk/ womens-health/clinical-guidance/management-hiv-pregnancy-green-top-39 Access on June $30^{\text {th }} 2012$.

[6] Forbes, J., Alimenti, A., Singer, J., Brophy, J., Bitnun, A., Sanson, L., et al. (2012). A national review of vertical HIV transmission. AIDS, 26(6).

[7] Blair, J. M., Hanson, D. L., Jones, J. L., et al. (2004). Trends in pregnancy rates among women with human immunodeficiency virus. Obstet Gynecol, 103, 663-668.

[8] M5 The European Mode of Delivery Collaboration. (1999). Elective caesarean-section versus vaginal delivery in prevention of vertical HIV-1 transmission: a randomized clinical trial. Lancet, 353, 1035-9.

[9] Coutsoudis, A., Pillay, K., Spooner, E., Kuhn, L., \& Coovadia, H. M. (1999). Influence of infant feeding patterns on early mother to child transmission of HIV-1 in Durban South Africa: A prospective cohort study. Lancet, 354(9177), 471-477.

[10] Melvin, Z., Alarcon, A. J., Velasquez, J. C., et al. (2004). Rapid HIV type 1 testing of women presenting in late pregnancy with unknown HIV status in Lima, Peru. Aids Research- Human retroviruses, 20(10), 1046-1052. 
[11] Connor, E. M., Sperling, R. S., \& Gelber, R. (1994). Reduction of maternal- infant transmission of human immunodeficiency virus type 1 with zidovudine treatment. $N$ Engl J Med, 331(18), 1173-80.

[12] Read, J., Cahn, P., Losso, M., Pinto, J., et al. (2007). Management of Human Immunodeficiency Virus-Infected Pregnant Women at Latin American and Caribbean Sites. Obstet Gynecol J, 109(6), 1358-1367.

[13] Valdés, E. (2002). VIH-SIDA y embarazo: actualización y realidad en Chile. Rev Chil Obstet Ginecol, 67, 160-6.

[14] Burns, D. N., Nourjah, P., Minkoff, H., Korelitz, J., Biggar, R. J., Landesman, S., Rubinstein, A., Wright, D., \& Nugent, R. P. (1996). Changes in CD4+ and CD8+ cell levels during pregnancy and post partum in women seropositive and seronegative for human immunodeficiency virus-1. Am J Obstet Gynecol, 174, 1461-8.

[15] Minkoff, H. L., Willoughby, A., Mendez, H., Moroso, G., Holman, S., Goedert, J. J., \& Landesman, S. H. (1990). Serious infections during pregnancy among women with advanced HIV infection. Am J Obstet Gynecol, 162, 30-4.

[16] Capítulo “VIH- SIDA y embarazo". (2006 ). E. Valdés. Libro “Urgencias y complicaciones en Obstetricia" M García Huidobro. J Hasbún. Editorial Mediterráneo, 447.

[17] “Mujer y VIH". (2002). E. Herane, E. Valdés. Libro SIDA. C. Sepúlveda A. Afani. Editorial Mediterráneo. Tercera edición, 721.

[18] French, R., \& Broklehurst, P. (1998). The effect of pregnancy on survival in women infected with HIV: a systematic review of the literature and meta-analysis. $\mathrm{Br}$. J Obstet Gynecol, 105, 827-35.

[19] Wade, A.S, et al. (2005). HIV infection and sexually transmitted infections among men who have sex with men in Senegal. AIDS, 19, 2133-2140.

[20] De Bruyn, M., \& Paxton, S. (2005). HIV testing of pregnant women-what is needed to protect positive women's needs and rights? Sex Health, 2, 143-51.

[21] Dhai, A., \& Noble, R. (2005). Ethical issues in HIV. Best Pract Res Clin Obstet Gynaecol, $19,255-67$.

[22] Bowden, FJ. (2005). Reconsidering HIV testing-consent is still the key. Sex Health, 2, 165-7.

[23] Chou, R., Smits, A. K., Huffman, L. H., Fu, R., \& Korthuis, P. T. (2005). US Preventive Services Task Force. Prenatal screening for HIV: A review of the evidence for the U.S. Preventive Services Task Force. Ann Intern Med, 143, 38-54.

[24] Campos-Outcalt, D. (2007). Time to revise your HIV testing routine. J Fam Pract, 56, 283-4.

[25] Winn, H., \& Hobbins, J. (2000). Clinical Maternal-Fetal Medicine. Parthenon Publish Group; Cap 26. 
[26] Abarzúa, F. (2012). VIH y Embarazo. Capítulo 21. pag Found in: www.acog.cl/educación.php?sort=ddAccess on June $30^{\text {th }} ., 413-23$.

[27] The European Collaborative Study. (1999). Maternal viral load and vertical transmission of HIV-1: an important factor but not the only one. AIDS, 13, 1377-1385.

[28] Khan, M., Pillay, T., Moodley, J. M., et al. (2001). Maternal mortality associated with tuberculosis-HIV-1 co-infection in Durban, South Africa. AIDS, 15, 1857-1863.

[29] Duri, K., Müller, F., Gumbo, F., Kurewa, N., Rusakaniko, S., Chirenje, M., et al. (2011). Human Immunodeficiency Virus (HIV) types Western blot (WB) band profiles as potential surrogate markers of HIV disease progression and predictors of vertical transmission in a cohort of infected but antiretroviral therapy naïve pregnant women in Harare, Zimbabwe. BMC infectious Diseases, 11, 7.

[30] El Beitune, P., Duarte, G., Quintana, S. M., Figueiro-Filho, E. A., Marcolin, A. C., \& Abduch, R. (2004). Antiretroviral therapy during pregnancy and early neonatal life: consequences for HIV-exposed uninfected children. Braz J Infect Dis, 8, 140-50.

[31] Use of antiretroviral drugs for treating pregnant women and preventing HIV infection in infants Programmatic update. (2012). Abril. Found in: http:// www.who.int/hiv/pub/mtct/programmatic_update2012/en/index.html Access on June 30th.

[32] Abarzúa, F., Pérez, C., Callejas, C., Yombi, J. C., \& Vandercam, B. (2004). Ausencia de transmisión perinatal de $\mathrm{VIH}$ en 40 embarazadas tratadas con terapia anti-retroviral de alta potencia. Rev Chil Obstet Ginecol, 69, 232-8.

[33] Abarzúa, F., Nuñez, F., Hubinont, C., Bernard, P., Yombi, J. C., \& Vandercam, B. (2005). Human immunodeficiency virus (HIV) infection in pregnancy: antiretroviral treatment (ART) and mode of delivery. Rev Chil Infectol, 22, 327-37.

[34] Guía Clínica (2010). Síndrome de Inmunodeficiencia Adquirida. VIH/SIDA. Found in : www.redsalud.gov.cl/portal/url/item/7220fdc4340c44a9e04001011f0113b9.pdf Access on June $30^{\text {th }} 2012$

[35] Poirier, M. C., Olivero, O. A., Walker, D. M., \& Walker, V. E. (2004). Perinatal genotoxicity and carcinogenicity of anti-retroviral nucleoside analog drugs. Toxicol Appl Pharmacol, 199(2), 151-61.

[36] Brogly, S. B., Ylitalo, N., Mofenson, L. M., Oleske, J., Van Dyke, R., Crain, M. J., Abzug, M. J., Brady, M., Jean-Philippe, P., Hughes, M. D., \& Seage, G. R. 3rd. (2007). In utero nucleoside reverse transcriptase inhibitor exposure and signs of possible mitochondrial dysfunction in HIV-uninfected children. AIDS, 21, 929-38.

[37] Lorenzini, P., Spicher, V. M., Laubereau, B., Hirschel, B., Kind, C., Rudin, C, et al. (1998). Antiretroviral therapies in pregnancy: maternal, fetal and neonatal effects. Swiss HIV Cohort Study, the Swiss Collaborative HIV and Pregnancy study, and the Swiss Neonatal AHIV study. AIDS, 12(18), F241-7. 
[38] Read, J. S., \& Newell, M. K. (2005). Efficacy and safety of cesarean delivery for prevention of mother-to-child transmission of HIV-1. Cochrane Database Syst Rev [4], CD005479.

[39] Safety and Toxicity of individual Antiretroviral Agents in pregnancy. (2012). Found in: http://img.thebody.com/hivatis/pdfs/pregnancy_guide.pdf Access on June $30^{\text {th }}$.

[40] Semprini, A. E., Vucetich, A., \& Hollander, L. (2004). Sperm washing, use of HAART and role of elective caesarean section. Curr Opin Obstet Gynecol, 16, 465-70. 